\title{
Framingham versus PROCAM para establecer el riesgo cardiovascular. ¿Existe diferencia?
}

Framingham versus PROCAM to establish cardiovascular risk. Is there a difference?

a

Auquilla Pauta Katherine Estefanía', Cabrera Capelo Adriana Jackeline ${ }^{2}$, Sacoto Molina Adrián Marcelo ${ }^{3}$

VOLUMEN 38 | N² 2 | AGOSTO 2020

FECHA DE RECEPCIÓN: 13/01/2020 FECHA PUBLICACIÓN: 31/08/2020 FECHA DE REVISIÓN: 27/12/2020 FECHA DE REEDICIÓN: 15/03/2021

1. Ministerio de Salud Pública. Centro de Salud El Pan. Cuenca - Ecuador

2. Ministerio de Salud Pública. OCP. Cuenca - Ecuador

3. Universidad de Cuenca. Facultad de Ciencias Médicas. Docente de la carrera de Medicina. Cuenca - Ecuador

\section{Artículo Original Original Article}

DOI: https://doi.org/10.18537/RFCM.38.02.01

Correspondencia:

estefiauquilla@gmail.com

Dirección:

Parroquia Paccha, barrio Unión y

Progreso

Código Postal:

010160

Teléfono:

072879524 - 0983858793

Cuenca - Ecuador

\section{RESUMEN}

Introducción: las enfermedades cardiovasculares son la principal causa de muerte a nivel mundial, existen escalas que permiten determinar el riesgo de padecerlas, de ahí la importancia de estudiar su comportamiento en nuestra población.

Objetivo: determinar la concordancia de las escalas de riesgo cardiovascular Framingham y PROCAM.

Materiales y métodos: se realizó un estudio de tipo analítico con 500 pacientes con edades entre 35 y 65 años que cumplían con los criterios de inclusión, los datos se tomaron de las historias clínicas de consulta externa de Medicina Interna del Hospital José Carrasco Arteaga, centro de atención de tercer nivel, en el periodo enerodiciembre 2016. La concordancia se estableció a través del índice kappa de Cohen y el método de Bland y Altman.

Resultados: el índice kappa calculado entre las escalas de riesgo cardiovascular Framingham y PROCAM es de 0.5246; ( $p<0.0001)$.

Conclusión: la concordancia hallada entre Framingham y PROCAM es moderada. De acuerdo al gráfico de Bland y Altman las escalas son más concordantes en valores bajos de riesgo cardiovascular.

Palabras claves: factores de riesgo, enfermedad cardiovascular, anomalías cardiovasculares. 


\section{ABSTRACT \\ Introduction: cardiovascular diseases are the main cause of death worldwide, there are scales that allow determining the risk of suffering, hence the importance of studying their behavior in our population.}

Objective: to determine the concordance of the Framingham and PROCAM cardiovascular risk scales.

Materials and methods: an analytical study was carried out with 500 patients between the ages of 35 and 65 who met the inclusion criteria, the data was taken from the clinical records of the outpatient clinic of Internal Medicine of the Hospital José Carrasco Arteaga, center thirdlevel care, in the period January-December 2016. The agreement was established through the Cohen kappa index and the Bland and Altman method.

Results: the kappa index calculated between the Framingham and PROCAM cardiovascular risk scales is 0.5246; $(p<0.0001)$.

Conclusion: the agreement found between Framingham and PROCAM is moderate. According to the Bland and Altman graph, the scales are more concordant in low values of cardiovascular risk.

Key words: risk factors, cardiovascular disease, cardiovascular abnormalities.

\section{INTRODUCCIÓN}

Las enfermedades crónicas no transmisibles constituyen una de las principales causas de mortalidad en nuestro medio, las más frecuentes son la cardiopatía isquémica y eventos cerebrovasculares. En el Ecuador el patrón se repite dado que según los datos del Instituto Nacional de Estadísticas y Censos
(INEC), en el año 2014 se reportaron 4430 muertes por enfermedades isquémicas del corazón y 3777 muertes por enfermedades cerebrovasculares [1].

Varios autores han desarrollado fórmulas que permiten calcular el riesgo cardiovascular, que se relaciona con la probabilidad de un individuo de presentar un trastorno cardiovascular durante un periodo de tiempo $[2,3]$.

Se aplicaron las escalas de riesgo cardiovascular Framingham y PROCAM (Estudio Prospectivo Cardiovascular Münster) y se determinó la concordancia que existe entre ellas. Los algoritmos de Framingham valoran la probabilidad de presentar eventos clínicos de enfermedad cardiovascular en personas que no las han padecido previamente [4], mientras que la calculadora PROCAM estima el riesgo de desarrollar enfermedad coronaria, mediante la función de Weibull y a su vez valora el riesgo de sufrir eventos cerebrovasculares isquémicos fatales y no fatales, utilizando la función de Cox [5].

El riesgo cardiovascular varía de acuerdo a algunas situaciones: la población en estudio, las diferencias genéticas, los comportamientos de salud y factores sociales propios de cada localidad [6,7]; en Colombia durante el año 2014, se desarrolló una validación y calibración de los modelos Framingham y PROCAM, en este se recomienda multiplicar la estimación obtenida en Framingham por 0.75 y en PROCAM por 0.93 , se pudo notar que dado el pequeño número de pacientes en categoría de riesgo alto el modelo no estaba calibrado para esta categoría a razón de la gran diferencia absoluta entre eventos esperados y observados $[8,9]$.

Una gran variabilidad de resultados caracteriza a los estudios de concordancia entre las escalas Framingham y PROCAM en distintos países y 
poblaciones, en los que dicha relación ha sido estudiada. La investigación acerca del tema más cercana a nuestro medio fue la del trabajo colombiano mencionado anteriormente; de ahí la importancia de desarrollar trabajos de investigación en nuestra población.

\section{MATERIALES Y MÉTODOS}

Se realizó un estudio de tipo analítico de correlación en el cual se consideraron como criterios de inclusión a las historias clínicas de pacientes entre 35 y 65 años de edad, que mostraban las variables necesarias para aplicar las escalas: edad, sexo, diabetes mellitus, tabaquismo, antecedentes familiares de enfermedad cardiovascular precoz, presión arterial sistólica y diastólica, colesterol total, colesterol HDL (High density cholesterol), colesterol LDL (Low density cholesterol) y triglicéridos. Fueron excluidas de este trabajo aquellas a las que el sistema no permitía acceder por pérdida de información u otra causa y las historias con datos incompletos.

Constituyeron el universo del estudio las 14,333 historias clínicas de los pacientes de consulta externa de medicina interna del Hospital José Carrasco Arteaga que acudieron a consulta durante el periodo comprendido entre el 1 de enero al 31 de diciembre del 2016. Posteriormente por conveniencia se escogieron 500 historias clínicas de pacientes que cumplían con los criterios de inclusión.

Para el registro y tabulación de los datos se usó: Excel 2013, PSPP Free Software Foundation, Inc. versión 1.0.1-g818227 y Epidat 4.1. La concordancia fue determinada mediante el índice kappa de Cohen y el método de Bland y Altman.

Para Framingham se empleó el algoritmo basado en el estudio Wilson, Prediction of
Coronary Heart Disease Using Risk Factor Categories, para pacientes entre 30 y 74 años que considera las variables edad, sexo, tabaquismo, diabetes mellitus, presión arterial sistólica, presión arterial diastólica, colesterol total, y colesterol HDL [10]. De acuerdo al riesgo los pacientes se clasifican en riesgo bajo: cuando es menor de 10\%, intermedio, cuando se halla entre $10 \%$ y $20 \%$, y alto si supera el 20\% [11]. Este algoritmo valora el riesgo de desarrollar enfermedad coronaria total como muerte de origen coronario, infarto de miocardio, angina de pecho o insuficiencia coronaria en los próximos 10 años [8].

En el caso de la escala de PROCAM se utilizó un modelo aplicado a hombres de entre $35 \mathrm{y}$ 65 años empleando las variables edad, sexo, menopausia, presencia o ausencia de diabetes mellitus o niveles de glucosa mayores o igual a $120 \mathrm{mg} / \mathrm{dl}$, tabaquismo, antecedentes familiares de infarto agudo de miocardio precoz, presión arterial sistólica, colesterol LDL, colesterol HDL y triglicéridos [8,12]. Los valores los ubican en riesgo bajo, si el puntaje es menor de 10\%, en intermedio, si está entre 10\% y 20\% y en alto si supera el $20 \%$ [13]. Con esta fórmula se estima el riesgo de desarrollar enfermedad coronaria en los próximos 10 años. Para las mujeres se aplica un factor de reducción de 0.25 si no existe diabetes mellitus [5].

Debemos acotar que los valores fueron corregidos de acuerdo al ajuste de Colombia (Framingham por 0.75 y en PROCAM por 0.93) [8].

\section{RESULTADOS}




\section{TABLA N 1}

Distribución de la población de estudio según características demográficas

\begin{tabular}{llrr}
\hline & & N & Características \\
& $35-39$ & 8 & 1.6 \\
& $40-44$ & 29 & 5.8 \\
\multirow{3}{*}{ Edad (años)* } & $45-49$ & 29 & 5.8 \\
& $50-54$ & 64 & 12.8 \\
& $55-59$ & 97 & 19.4 \\
Sexo & $60-65$ & 273 & 54.6 \\
\hline \multirow{3}{*}{ Estado civil } & Masculino & 250 & 50 \\
& Femenino & 250 & 50 \\
\hline \multirow{3}{*}{ Residencia } & Casado & 403 & 80.6 \\
& Soltero & 34 & 6.8 \\
& Divorciado & 36 & 7.2 \\
& Viudo & 18 & 3.6 \\
& Unión libre & 9 & 1.8 \\
\hline & Cuenca & 418 & 83.6 \\
& Machala & 21 & 4.2 \\
& Azogues & 13 & 2.6 \\
\hline
\end{tabular}

*Media: 54.80. Desvío estándar: 7.746

\section{TABLA N²}

Distribución de la población de estudio según el riesgo cardiovascular, aplicando las escalas Framingham y PROCAM ajustadas

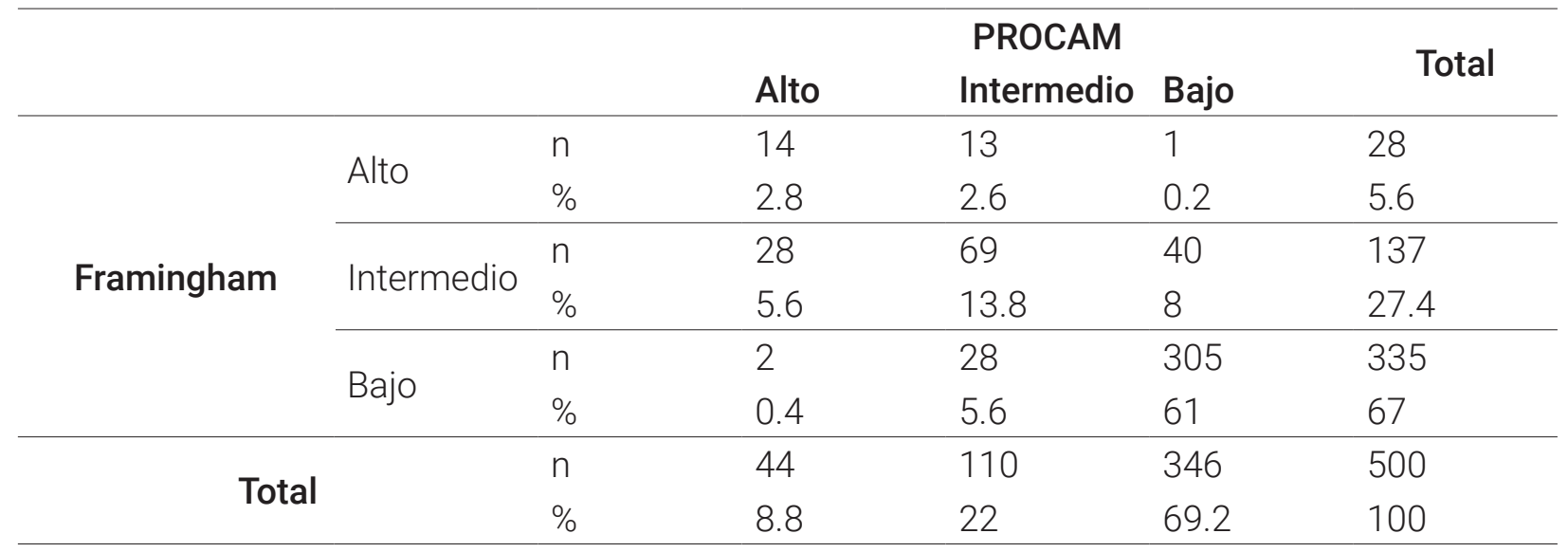




\section{TABLA N³}

Distribución de la población de estudio según riesgo cardiovascular y sexo, aplicando las escalas Framingham y PROCAM ajustadas

\begin{tabular}{|c|c|c|c|c|c|c|c|}
\hline & & & & & PROCAN & & Total $>$ \\
\hline & & & & Alto & Intermedio & Bajo & Iotal \\
\hline & & $\Delta+\theta_{0}$ & $n$ & 8 & 6 & 0 & 14 \\
\hline & & Alto & $\%$ & 3.2 & 2.4 & 0 & 5.6 \\
\hline Mnosding & Framinghom & Lotornodis & $\mathrm{n}$ & 18 & 40 & 19 & 77 \\
\hline Masculıno & Framıngham & Intermedı & $\%$ & 7.2 & 16 & 7.6 & 30.8 \\
\hline & & & $\mathrm{n}$ & 1 & 9 & 149 & 159 \\
\hline & & Вајо & $\%$ & 0.4 & 3.6 & 59.6 & 63.6 \\
\hline & Tot & & $N$ & 27 & 55 & 168 & 250 \\
\hline & lot & & $\%$ & 10.8 & 22 & 67.2 & 100 \\
\hline & & $\Delta+t_{0}$ & $\mathrm{n}$ & 6 & 7 & 1 & 14 \\
\hline & & Alto & $\%$ & 2.4 & 2.8 & 0.4 & 5.6 \\
\hline Comoning & Frominghom & Intormodis & $n$ & 10 & 29 & 21 & 60 \\
\hline remenino & rramingnam & imiermedio & $\%$ & 4.0 & 11.6 & 8.4 & 24 \\
\hline & & Rain & $n$ & 1 & 19 & 156 & 176 \\
\hline & & Вајо & $\%$ & 0.4 & 7.6 & 62.4 & 70.4 \\
\hline & & & $\mathrm{n}$ & 17 & 55 & 178 & 250 \\
\hline & Tot & & $\%$ & 6.8 & 22 & 71.2 & 100 \\
\hline
\end{tabular}

El grupo de estudio estuvo compuesto por un $50 \%$ de hombres y el $50 \%$ de mujeres, la media de edad fue de 54 años y el grupo con mayor número de participantes fue el de 60 a 65 años (Ver Tabla N¹). Al comparar los dos modelos de estratificación de riesgo, se observa que la escala Framingham incluye a más pacientes dentro de riesgo moderado, mientras que PROCAM clasifica un mayor número de casos dentro de riesgo bajo y alto. Estos modelos presentaron una concordancia del 2.8, 13.8 y $61 \%$, al momento de estratificar pacientes dentro de las categorías de alto, intermedio y bajo riesgo respectivamente (Ver Tabla N²). Este mismo análisis se realizó tras categorizar a la muestra de acuerdo al sexo, obteniéndose una mejor concordancia al clasificar pacientes dentro de riesgo alto e intermedio en la población masculina vs femenina; 3.2 vs $2.4 \%$ para riesgo alto; 16 vs $11.6 \%$ para riesgo intermedio y 59.6 vs $62.4 \%$ para riesgo bajo (Ver Tabla N³).

\section{TABLA N ${ }^{\circ} 4$}

Concordancia entre las escalas de riesgo cardiovascular Framingham y PROCAM ajustadas, utilizando el índice Kappa de Cohen, Cuenca 2018

\begin{tabular}{cccc}
\hline & Valor & Error estándar & Valor de P \\
\hline Kappa & 0.5246 & 0.0340 & $\mathrm{P}<0.0001$ \\
\hline
\end{tabular}




\section{TABLA N5}

Concordancia entre las escalas de riesgo cardiovascular Framingham y PROCAM ajustadas, utilizando el índice Kappa de Cohen, según sexo. Cuenca. 2018

\begin{tabular}{ccccc}
\hline Sexo & & Valor & Error estándar & Valor de P \\
\hline Masculino & Kappa & 0.5750 & 0.0455 & $\mathrm{P}<0.0001$ \\
\hline Femenino & Kappa & 0.4662 & 0.0533 & $\mathrm{P}<0.0001$ \\
\hline
\end{tabular}

Según la escala de Landis y Koch la y Koch tanto para la población masculina concordancia es moderada al obtenerse un como femenina la concordancia es moderada índice Kappa de 0.52; (0.41-0.60 moderado) a pesar de que el índice kappa es mayor en el (Ver Tabla N4), al aplicar la escala de Landis sexo masculino (Ver Tabla N5) [14].

Figura $\mathrm{N}^{\circ} 1$

Concordancia entre las escalas de riesgo cardiovascular Framingham y PROCAM ajustadas, de acuerdo al método de Bland y Altman.

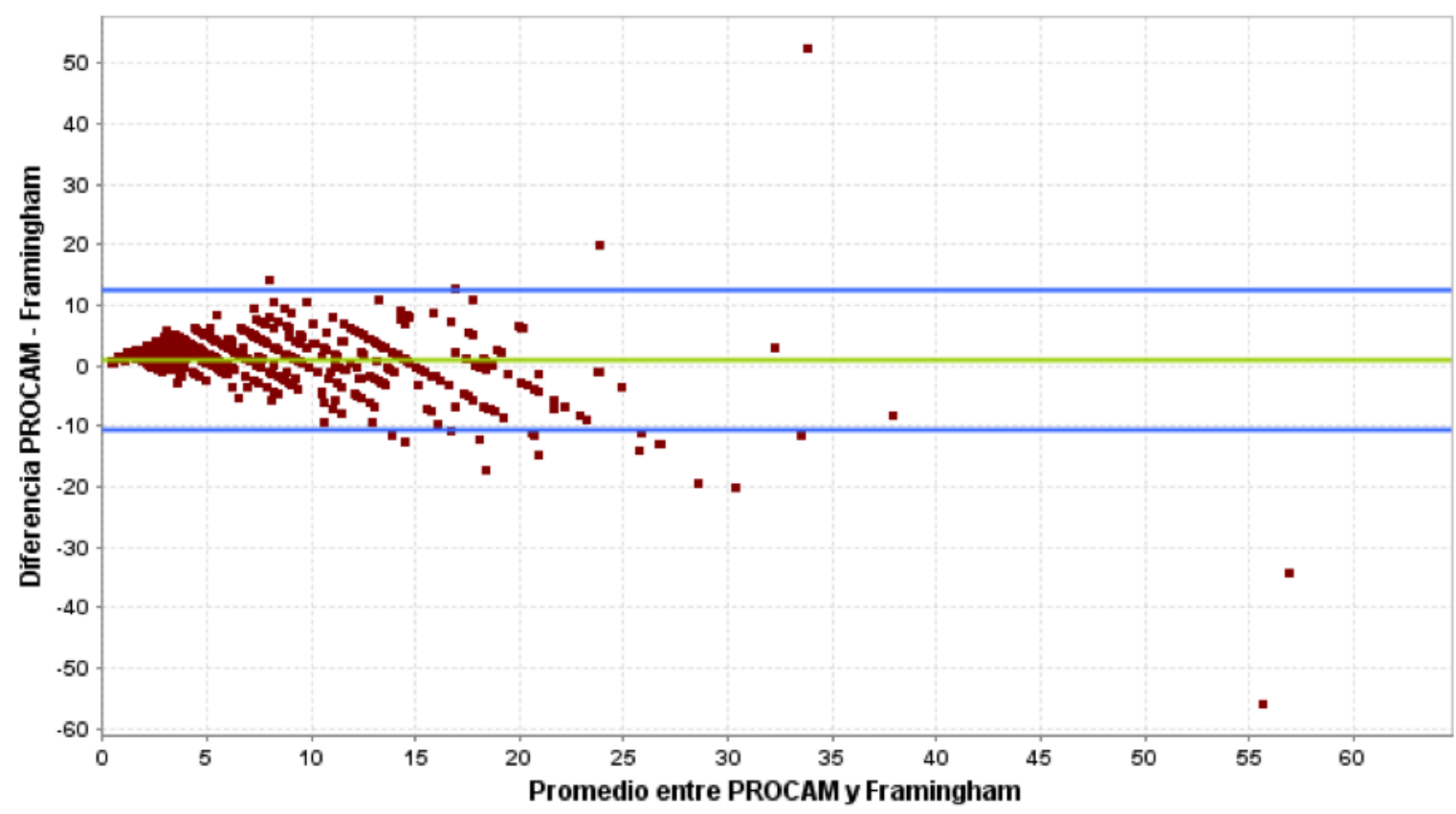

Línea verde: diferencia media $(0,82)$

Líneas azules: límites de concordancia (+/- 2 desvío estándar de la media: 12,37; - 10,73

En Figura $N^{\circ} 1$ se observa que la concordancia entre las escalas de Framingham y PROCAM disminuye conforme aumenta el riesgo cardiovascular, es así que son muy concordantes en el grupo de bajo riesgo.

\section{DISCUSIÓN}

La concordancia hallada entre las escalas de riesgo cardiovascular Framingham y PROCAM es moderada (kappa 0.5246; $p<0.0001$ ). De 
acuerdo al gráfico de Bland y Altman las escalas son menos concordantes conforme aumenta el riesgo cardiovascular. La concordancia tanto para el sexo femenino y masculino es moderada, a pesar de que el índice kappa fue mayor en este último grupo (kappa 0.4662 y 0.5750 respectivamente).

Para Knobel et al [15]. en su trabajo realizado en Barcelona, la concordancia entre las escalas de riesgo cardiovascular es aceptable (Kappa 0.36) siendo inferior a la encontrada en el presente estudio.

En investigaciones realizadas en Brasil la concordancia entre Framingham y PROCAM es moderada según Moreira et al [16], ya que se obtuvo un índice kappa de 0.433. Según Barros et al [17], la concordancia es moderada (kappa 0.51 - 0.59) cuando se compara las escalas en pacientes con síndrome metabólico, pero al excluir del estudio a las personas con este síndrome la concordancia aumenta, siendo esta buena (kappa 0.66 - 0.71). Los resultados hallados son similares a este estudio ya que no se excluyeron las historias clínicas de pacientes con síndrome metabólico.

En Lima según Pino et al [18], al estudiar a pacientes masculinos con VIH, el índice kappa obtenido es moderado (kappa 0.56), resultado semejante al obtenido cuando se excluye a la población femenina. Por otra parte, al observar el método de Bland y Altman se evidencia que la concordancia entre Framingham y PROCAM disminuye conforme aumenta el riesgo cardiovascular a intermedio y alto.

Álvarez et al. [19], en Asturias al analizar únicamente a la población masculina, encontró un índice kappa de 0.71 , siendo este superior al encontrado en este estudio.

Entre las limitaciones de este trabajo, se recalca que la información fue recolectada a través de historias clínicas previamente elaboradas. La bibliografía sobre el tema es escasa, la mayor parte de investigaciones son realizadas en poblaciones masculinas y en pacientes $\mathrm{VIH}$. En Ecuador no se han encontrado estudios previos que nos permitan comparar los resultados.

Por otra parte, las escalas de riesgo cardiovascular PROCAM y Framingham no han sidovalidadas enel país por loqueconsideramos aplicar los ajustes correspondientes a Colombia por ser la población más cercana y parecida a la nuestra (0.93 para PROCAM y 0.75 para Framingham) [8].

Recomendamos la realización de investigaciones que permitan la validación de PROCAM y Framingham en Ecuador para catalogar con mayor precisión a los pacientes. En caso de no ser posible la validación, sugerimos aplicar los ajustes de Colombia.

\section{CONCLUSIONES}

La concordancia entre las escalas de riesgo cardiovascular PROCAM y Framingham es moderada (kappa 0.5246). Esta disminuye conforme aumenta el riesgo cardiovascular, debido a que el ajuste de Colombia no fue calibrado para riesgo alto.

El modelo PROCAM minimiza el riesgo en riesgos bajos y sobrevalora en riesgos altos al comparar con Framingham por categorías (69.2\% vs $67 \%$; $8.8 \%$ vs $5.6 \%)$

Al contrastar los modelos de riesgo cardiovascular el índice kappa es superior en la población masculina (kappa: 0.575 vs 0.466) en virtud de que PROCAM fue planteada primariamente para varones.

Por lo tanto, las escalas Framingham y PROCAM deben ser utilizadas con prudencia 
y teniendo en cuenta otros criterios clínicos ya que su concordancia no es buena.

\section{ASPECTOS BIOÉTICOS}

El protocolo fue entregado a la Comisión de Proyectos de Investigación, donde fue aprobado por el comité de Bioética y por el Consejo Directivo de la Facultad de Ciencias Médicas de la Universidad de Cuenca. Posteriormente se obtuvo la autorización por parte del Coordinador General de Investigación del Hospital José Carrasco Arteaga.

Toda la información recolectada se guardó con absoluta confidencialidad, para ello se trabajó con el número de historia clínica de los pacientes

\section{INFORMACIÓN DE LOS AUTORES}

Auquilla Pauta Katherine Estefanía. Médica. Ministerio de Salud Pública. Centro de Salud El Pan. Cuenca-Azuay-Ecuador.

e-mail: estefiauquilla@gmail.com

ORCID:https://orcid.org/0000-0002-4186-1240

Cabrera Capelo Adriana Jackeline. Médica. Ministerio de Salud Pública. OCP. CuencaAzuay-Ecuador.

e-mail: adrijack209@gmail.com

ORCID:https://orcid.org/0000-0001-5007-6618

Sacoto Molina Adrián Marcelo. Médico. Magister en Investigación de la Salud. Universidad de Cuenca. Facultad de Ciencias Médicas. Carrera de Medicina. Cuenca-AzuayEcuador.

e-mail: adrian.sacoto@ucuenca.edu.ec ORCID:https://orcid.org/0000-0002-4048-9942

\section{CONTRIBUCIÒN DE LOS AUTORES:}

AK, CA: concepción y diseño del estudio, recolección de datos, análisis estadístico y la redacción del presente artículo.
SA: concepción y diseño del estudio, análisis estadístico y revisión del presente artículo.

\section{CONFLICTO DE INTERESES}

El presente estudio no presenta conflictos de interés.

\section{FUENTES DE FINANCIAMIENTO}

Esta investigación fue financiada por los propios autores.

\section{REFERENCIAS BIBLIOGRÁFICAS}

1. Instituto Nacional de Estadísticas y Censos del Ecuador INEC. Anuario de estadísticas vitalesnacimientosydefunciones. [Internet]. Ecuador en cifras. 2014. Disponible en: http://www.ecuadorencifras.gob.ec/ documentos/web-inec/Poblacion_y_ Demografia/Nacimientos_Defunciones/ Publicaciones/Anuario_Nacimientos_y_ Defunciones_2014.pdf

2. Hajar R. Framingham Contribution to Cardiovascular Disease. Heart Views Off J Gulf Heart Assoc. 2016;17(2):78-81. DOI: 10.4103/1995-705X.185130

3. Mahmood SS, Levy D, Vasan RS, Wang TJ. The Framingham Heart Study and the epidemiology of cardiovascular disease: a historical perspective. The Lancet. 2014 ;383(9921):999-1008. DOI: 10.1016/ S0140-6736(13)61752-3

4. D’Agostino Sr RB, Grundy S, Sullivan LM, Wilson P, Group CRP. Validation of the Framingham coronary heart disease prediction scores: results of a multiple ethnic groups investigation. Jama. 2001;286(2):180-187. DOI: 10.1001/ jama.286.2.180

5. Ruiz JS. Modelos europeos: PROCAM 
y SCORE. En: Control Global del Riesgo Cardiometabólico La disfunción endotelial como diana preferencial. Madrid: Díaz de Santos; 2012.

6. Wallace ML, Ricco JA, Barrett B. Screening Strategies for Cardiovascular Disease in Asymptomatic Adults. Prim Care. 2014;41(2):371-97. DOI: 10.1016/j. pop.2014.02.010

7. Nery MW, Martelli CMT, Aparecida Silveira E, Sousa CA de, Falco M de O, Castro A de CO de, et al. Cardiovascular risk assessment: $A$ comparison of the Framingham, PROCAM, and DAD equations in HIV-infected persons. Sci World J. 2013;2013:1-9. DOI: $10.1155 / 2013 / 969281$

8. Muñoz OM, Rodríguez NI, Ruiz Á, Rondón M. Validación de los modelos de predicción de Framingham y PROCAM como estimadores del riesgo cardiovascular en una población colombiana. Rev Colomb Cardiol. 2014;21(4):202-12. DOI:10.1016/j. rccar.2014.02.001

9. Muñoz $\vee$ OM, Ruiz Morales ÁJ, Mariño Correa A, Bustos C. MM. Concordancia entre los modelos de SCORE y Framingham y las ecuaciones AHA/ACC como evaluadores de riesgo cardiovascular. Rev Colomb Cardiol. 2017;24(2):110-6. DOI: 10.1016/j. rccar.2016.06.013

10. Wilson PW, D’Agostino RB, Levy D, Belanger AM, Silbershatz $H$, Kannel WB. Prediction of coronary heart disease using risk factor categories. Circulation. 1998;97(18):183747. DOI: 10.1161/01.CIR.97.18.1837

11. D'Agostino RB, Russell MW, Huse DM, Ellison RC, Silbershatz $H$, Wilson PW, et al. Primary and subsequent coronary risk appraisal: new results from the Framingham study. Am Heart J. 2000;139(2):272-81. DOI: 10.1016/S0002-8703(00)90236-9

12. Linares JMM, García JM. Comparación de las calculadoras online de riesgo cardiovascular. Enferm Cardiol. 2016;66-70. Disponible en: https://www. enfermeriaencardiologia.com/wp-content/ uploads/67_03.pdf

13. Tolunay $H$, Kurmus O. Comparison of coronary risk scoring systems to predict the severity of coronary artery disease using the SYNTAX score. Cardiol J. 2016;23(1):51-6. DOI: 10.5603/CJ.a2015.0074

14. Landis JR, Koch GG. The Measurement of Observer Agreement for Categorical Data. Biometrics. 1977;33(1):159. DOI: $10.2307 / 2529310$

15. KnobelH, Jericó C,MonteroM, SorliML, Velat M, Guelar A, et al. Global Cardiovascular Risk in Patients with HIV Infection: Concordance and Differences in Estimates According to Three Risk Equations (Framingham, SCORE, and PROCAM). AIDS Patient Care STDs. 2007;21(7):452-7. DOI: 10.1089/ apc. 2006.0165

16. Moreira Guimarães MM, Bartolomeu Greco D, Ingles Garces á. H, de Oliveira AR, Bastos Fóscolo R, de Campos Machado LJ. Coronary heart disease risk assessment in HIV-infected patients: a comparison of Framingham, PROCAM and SCORE risk assessment functions. Int $\mathrm{J}$ Clin Pract. 2010;64(6):739-45. DOI: 10.1111/j.17421241.2009.02248.x

17. BarrosZM, deAlencarXimenes RA, MirandaFilho DB, de Albuquerque M de FPM, Melo HRL, Carvalho ÉH, et al. Comparison Between the Framingham and Prospective Cardiovascular of Münster Scores for Risk 
Assessment of Coronary Heart Disease in Human Immunodeficiency Virus-Positive Patients in Pernambuco, Brazil. Metab Syndr Relat Disord. 2010;8(6):489-97. DOI: 10.1089/met.2009.0100

18. Lister-Del Pino P, León-Amenero G, LeivaMontejo A, Segura ER. Concordance between Procam and Framingham cardiovascular risk scores among men receiving HIV treatment at a National Hospital in Lima, Peru 2013. Rev Peru Med Exp Salud Publica. 2015;32(4):731-8. PMID: 26732922

19. Álvarez $A$, López $V$, Prieto $M$, Díaz L, Herrero P, Vázquez J, et al. PROCAM y Framingham por categorías: ¿miden igual riesgo? MEDIFAM. 2002;12:2605. Disponible en: http://scielo.isciii.es/ scielo.php?script=sci_arttext\&pid=S1131$57682002000400003 \&$ lng $=e s \& n r m=i s o \& t \mid$ $\mathrm{ng}=\mathrm{es}$ 